\title{
Similarities in the temporal properties of gamma-ray bursts and soft gamma-ray repeaters
}

\author{
S. McBreen, L. Moran, B. McBreen, L. Hanlon, \\ J. French, M. Conway \\ Department of Experimental Physics, University College Dublin, Dublin 4, Ireland.
}

\begin{abstract}
Magnetars are modelled as sources that derive their output from magnetic energy that substantially exceeds their rotational energy [1]. An implication of the recent polarization measurement of GRB 021206 is that the emission mechanism may be dominated by a magnetic field that originates in the central engine [2]. Similarities in the temporal properties of SGRs and GRBs are considered in light of the fact that the central engine in GRBs may be magnetically dominated. The results show that 1) the time intervals between outbursts in SRG 1806-20 and pulses in GRBs are consistent with lognormal distributions and 2) the cumulative outputs of SGRs and GRBs increase linearly with time. This behaviour can be successfully modelled by a relaxation system that maintains a steady state situation.
\end{abstract}

\section{INTRODUCTION}

Over the past decade the evidence for neutron stars with ultra-strong magnetic fields or magnetars has become convincing. Soft gamma-ray repeaters (SGRs) are associated with supernova remnants and have multiple bursts of gamma-rays which distinguish them from gamma-ray bursts (GRBs) e.g. [3, 4]. SGRs have intensely active periods which can last for weeks or months that are separated by quiescent phases lasting for years or decades [5]. The most intense outburst recorded to date has $\sim 10^{44}$ ergs in $\gamma$ rays. Several SGRs and anomalous X-ray pulsars have been found to be X-ray pulsars that have unusually high spin down rates. The rapid reduction in spin is usually attributed to magnetic breaking caused by the super-strong magnetic fields with values above $10^{14}$ G. In the magnetar model the magnetic field provides the burst energy [1]. A common scenario is that stresses build up in the magnetic field and cause a quake in the crust of the neutron star which ejects plasma Alfvèn waves through the magnetosphere.

GRBs are non-recurrent catastrophic events that radiate $\sim 10^{51} \mathrm{ergs}$ in $\gamma$-rays. There is substantial evidence that GRBs are linked to supernova explosions e.g. [6]. They are often modelled as accretion onto a newly formed black hole e.g. [7]. Recent measurements suggest that the $\gamma$-rays are highly polarised by the magnetic field which may originate in the central engine with values of $B$ above $10^{17} \mathrm{G}[2]$. It is therefore interesting to compare the pulse properties of SGRs and GRBs. It has been noted that the lognormal distributions apply to the pulse properties of SGRs [8, 9, 10] and GRBs [11, 12, 13]. Furthermore the radio afterglow from the giant flare from SGR $1900+14$ is similar to radio afterglows from GRBs [14]. 


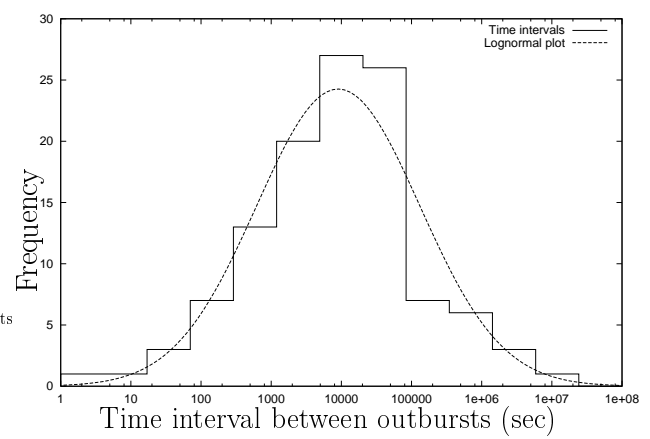

FIGURE 1. Histogram of the number of bursts from SGR 1806-20 versus time interval between the bursts. The dashed curve is a lognormal fit to the data. The SGR was observed by the International Cometary Explorer (ICE) and the data are taken from Ulmer et al [16].
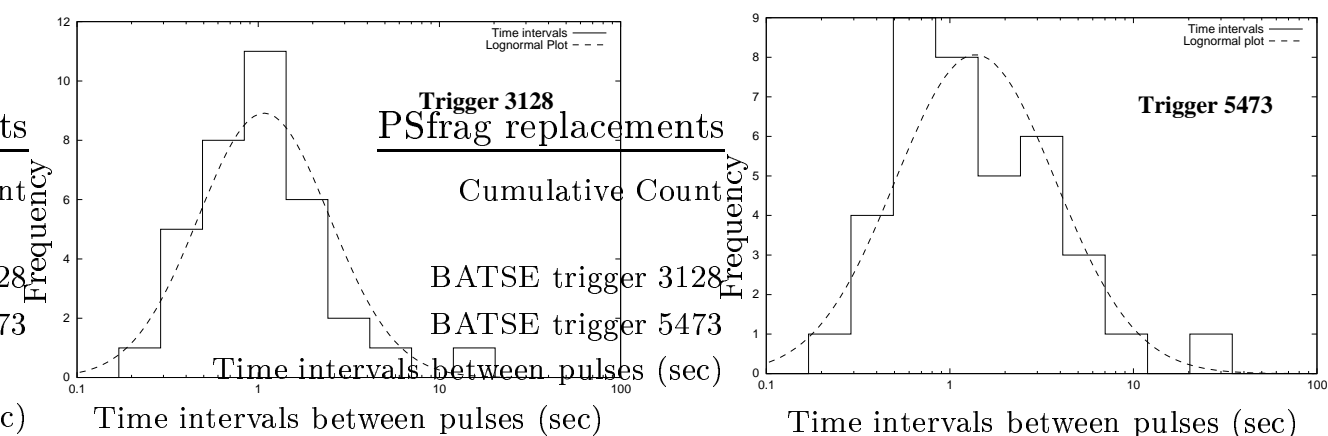

FIGURE 2. Histograms of the time interval between the pulses and lognormal representations for BATSE triggers GRB 940817 and GRB 960524 with 37 and 39 pulses respectively.

\section{TIME INTERVALS BETWEEN OUTBURSTS IN SGRS AND GRBS}

SGR 1806 -20 had a very active phase during 1983 when more than 100 outbursts were recorded [15, 16, 8]. The distribution of the time intervals between the outbursts is given in Figure 1 along with a lognormal fit to the data. Long time intervals between active phases ( $>10$ years) can easily be accomodated by the long tail of the lognormal distribution [17]. The time intervals between pulses in two BATSE GRBs with large numbers of pulses are given in Figure 2 along with lognormal plots. The pulse properties and time intervals between the pulses in short and long GRBs have been found to be lognormally distributed [11, 12, 18, 13, 19, 20]. It is interesting to note that the time intervals between radio glitches in the Vela pulsar are lognormally distributed [8].

In Fig. 1 the median time interval between SGR outbursts is $\sim 10^{4} \mathrm{~s}$ and in Fig. 2 the time interval between pulses in the GRBs is $\sim 1 \mathrm{~s}$.

\section{CUMULATIVE LIGHT CURVES FOR SGRS AND GRBS}

The cumulative light curve has been shown to be an interesting parameter for SGRs [21] and GRBs [22]. The running and cumulative light curves for the outbursts from SGR 


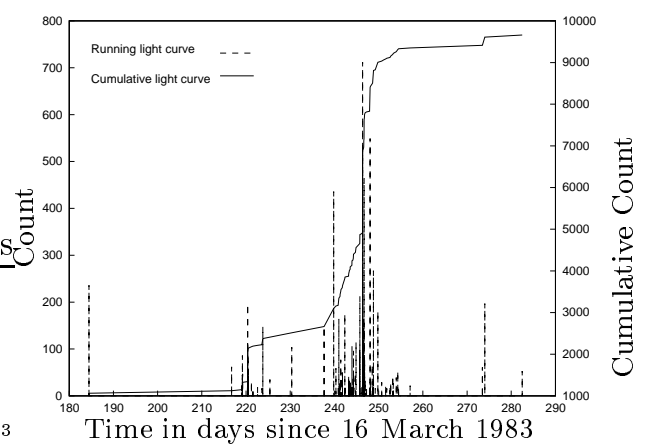

FIGURE 3. The running (dashed line) and cumulative (solid line) lightcurves of the outbursts from SGR $1806-20$ in 1983. The presentation is adapted from Palmer [21]. The cumulative output approximates to straight lines of different slopes in the active region in late 1983.
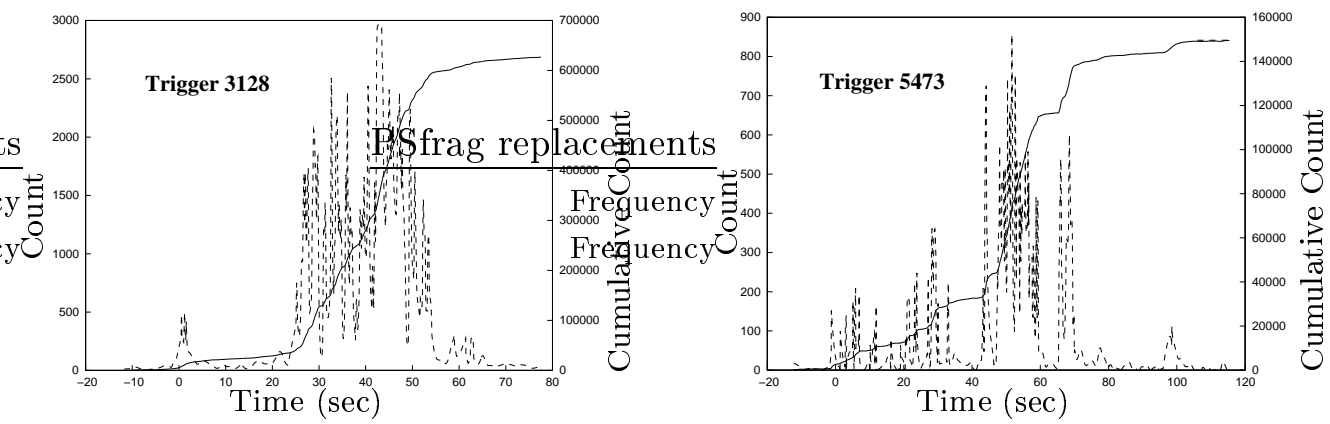

FIGURE 4. The running light curves (dashed line) and cumulative light curves (solid line) of GRB 940817 and GRB 960524 The cumulative light curves are approximately linear over the majority of the burst. The cumulative light curve of trigger 5473 can be fit by two linear sections. Trigger 3128 can be fit by one straight line despite the temporal complexity.

1806 - 20 are given in Figure 3. The cumulative light curves of a large majority of short and long GRBs has been found to be approximately linear with time. The running and cumulative light curves of the two GRBs in Figure 2 are given in Figure 4, The cumulative output increases linearly with time over the most active part of the GRB.

The cumulative output of the large majority of long and short GRBs was found to increase linearly with time [22].

\section{RELAXATION SYSTEMS}

The behaviour of bursting sources can be usefully compared with relaxation systems [21, 22]. A relaxation system is taken to be one that continuously accumulates energy from some process and discontinuously releases it. The energy in the reservoir at any time $t$ is

$$
E(t)=E_{o}+\int_{o}^{t} R(t) d t-\Sigma S_{i}
$$

where $E_{o}$ is the energy stored in the reservoir that accumulates energy at a rate $R(t)$ and discontinuously releases events of size $S_{i}$. 
The simplest system is referred to as a relaxation oscillator where there is a fixed level or trip-point that triggers a release of the energy when $E=E_{\max }$. The $S G R$ and GRB pulses are not consistent with this type of oscillator. More complicated behaviour occurs when the accumulation rate, trigger rate or release strength are not constant [23]. If the system starts from a minimum level $\mathrm{E}=\mathrm{E}_{\min }$, accumulates energy at a constant rate $\mathrm{R}=\mathrm{r}$, the sum of the releases is approximately a linear function of time i.e. $\Sigma S_{i} \propto r t$. This model can account for the approximately linear increase in cumulative counts. The output from GRBs and SGRs has a tendency to keep the cumulative count close to a linear function and maintain a steady state situation

In a minority of bright GRBs with many pulses it was found that the cumulative output increases with time as $\mathrm{t}^{2}$ in $\sim 10 \%$ of bursts and as $(1-\mathrm{t})^{2}$ in $\sim 5 \%$. This behaviour was attributed to a change in spin of the newly formed black hole [24].

\section{ACKNOWLEDGEMENT}

SMcB acknowledges IRCSET grant number RS/2002/820-8M for support. LH, BMcB and JF thank IRCSET Basic Research Grant number SC-2002-377 for support.

\section{REFERENCES}

1. Duncan, R., and Thompson, C., ApJ, 392 (1992).

2. Coburn, W., and Boggs, S. E., Nature, 423, 415-417 (2003).

3. Zhang, B., and Meszaros, P. (2003), |astro-ph/0311321|.

4. Dar, A., and De Rujula, A. (2003), |astro-ph/0308248|.

5. Mazets, E. P., Golentskii, S. V., Ilinskii, V. N., Aptekar, R. L., and Guryan, I. A., Nature, 282, 587589 (1979).

6. Hjorth, J. et al. , Nature, 423, 847-850 (2003).

7. MacFadyen, A. I., and Woosley, S. E., ApJ, 524, 262-289 (1999).

8. Hurley, K. J., McBreen, B., Rabbette, M., and Steel, S., A\&A, 288, L49-L52 (1994).

9. Gögüus, E., Woods, P. M., Kouveliotou, C., van Paradijs, J., Briggs, M. S., Duncan, R. C., and Thompson, C., ApJ, 526, L93-L96 (1999).

10. Gögüs, E., Woods, P. M., Kouveliotou, C., van Paradijs, J., Briggs, M. S., Duncan, R. C., and Thompson, C., ApJ, 532, L121-L124 (2000).

11. McBreen, B., Hurley, K. J., Long, R., and Metcalfe, L., MNRAS, 271, 662 (1994).

12. Li, H., and Fenimore, E. E., ApJ, 469, L115 (1996).

13. McBreen, S., Quilligan, F., McBreen, B., Hanlon, L., and Watson, D., A\&A, 380, L31 (2001).

14. Cheng, K. S., and Wang, X. Y., ApJ, 593, L85-L88 (2003).

15. Laros, J. G. et al., ApJ, 320, L111 (1987).

16. Ulmer, A., Fenimore, E. E., Epstein, R. I., Ho, C., Klebesadel, R. W., Laros, J. G., and Delgado, F., ApJ, 418, 395 (1993).

17. Hurley, K. J., McBreen, B., Delaney, M., and Britton, A., Ap\&SS, 231, 81-84 (1995).

18. Gupta, V., Das Gupta, P., and Bhat, P. N., "AIP Conf. Proc. 526: Gamma-ray Bursts, 5th Huntsville Symposium. Edited by R.M. Kippen et al.," 2000, p. 215.

19. Quilligan, F., McBreen, B., Hanlon, L., McBreen, S., Hurley, K. J., and Watson, D., A\&A, 385, 377-398 (2002).

20. Nakar, E., and Piran, T., MNRAS, 331, 40-44 (2002).

21. Palmer, D. M., ApJ, 512, L113-L116 (1999).

22. McBreen, S., McBreen, B., Hanlon, L., and Quilligan, F., A\&A, 393, L29-L32 (2002).

23. Ramirez-Ruiz, E., and Merloni, A., MNRAS, 320, L25 (2001).

24. McBreen, S., McBreen, B., Hanlon, L., and Quilligan, F., A\&A, 393, L15-L19 (2002). 